Seventh Meeting, 14th June 1901.

J. W. Butrers, Esq., M.A., B.Sc., President, in the Chair.

\title{
An Extension of Abel's Theorem on the Continuity of a Power Series.
}

\section{By Professor Grbson.}

When the series

$$
s=a_{0}+a_{1}+\ldots \ldots+a_{n}+\ldots \ldots . .
$$

is convergent, then Abel's Theorem shows that

Series of the form

$$
\text { if } f(x)=\sum a_{m} x^{m}, \underset{x=1}{\text { Limit }} f(x)=\Sigma a_{m}=s .
$$

$$
f(t)=\sum_{0}^{\infty} a_{m} e^{-a_{m} t}
$$

are of frequent occurrence in the Analytical Theory of Heat and in other branches of Mathematical Physics, and the conditions to which $f^{\prime}(t)$ is subject usually require that as $t$ tends to zero the function $f(t)$ should tend to the value $s$.

The necessity for the proof of Abel's Theorem may be shown by the consideration of particular cases. Thus the two functions, defined by series,

$$
\begin{aligned}
& f_{1}(x)=\sum_{m=1}^{\infty}(-1)^{m-1} \frac{x^{m}}{m} \\
& f_{2}(x)=\sum_{m=1}^{\infty}\left(\frac{x^{4 m-3}}{4 m-3}+\frac{x^{4 m-1}}{4 m-1}-\frac{x^{2 m}}{2 m}\right)
\end{aligned}
$$

are each equal to $\log (1+x)$ for the range $-1<x<1$. Hence by the very definition of a limit

$$
\operatorname{Limit}_{x=1} f_{1}(x)=\log 2=\operatorname{Limit}_{x=1} f_{2}(x) .
$$

The value $f_{1}(1)$ is $\log 2$; but the value $f_{2}(1)$ is $\frac{3}{2} \log 2$ and

$$
\operatorname{Limit}_{z=1} f_{2}(x) \neq \sum_{m=1}^{\infty}\left(\frac{1}{4 m-3}+\frac{1}{4 m-1}-\frac{1}{2 m}\right) \text {. }
$$


So far as I know, not much attention has been paid to series of the form (2), though the corresponding limit theorems for definite integrals are of course well known. It is obvious, however, that the method that applies to Abel's Theorem can be extended to many other cases, and on account of the frequent occurrence of these series in mathematical physics, it may not be out of place to refer to some of these cases.

Suppose then that $f(t)$ is defined by equation (2), the series $\Sigma a_{m}$ being convergent and having $s$ as its sum.

First, let $a_{1}, a_{2}, a_{3} \ldots .$. be an infinite increasing sequence of positive numbers having an upper limit $a$, that is

$$
0<a_{n_{2}}<a_{m+1}<a \text { for every integer } m \text {; }
$$

then, manifestly $f(t)$ is a continuous function for every positive value of $t$. We have to prove

$$
\underset{t=0}{\operatorname{Limit}} f(t)=\sum_{0}^{\infty} a_{m}=s
$$

When $\alpha_{m}$ is a positive integer for every value of $m$, we of course have Abel's Theorem $\left(e^{-t}=x\right)$.

Let

$$
f(t)=\phi(t)+\psi(t)
$$

where

$$
\begin{aligned}
& \phi(t)=\sum_{0}^{n} a_{m} e^{-a_{m} t} \\
& \psi(t)=\sum_{n+1}^{\infty} a_{m} e^{-a_{m} t}=\operatorname{Limit}_{p=\infty} \mathbf{R}_{n}
\end{aligned}
$$

where ${ }_{p} \mathbf{R}_{n}=a_{n+1} e^{-a_{n+1} t}+a_{n+2} e^{-a_{n+2} t}+\ldots \ldots+a_{n+p} e^{-a_{n+p} t}$.

Let $s_{n}=\sum_{0}^{n} a_{m}$ so that

then

$$
a_{n+1}=s_{n+1}-s_{n}, a_{n+2}=s_{n+2}-s_{n+1}, \ldots \ldots \ldots
$$

$$
\begin{aligned}
{ }_{p} \mathrm{R}_{n} & =\left(s_{n+1}-s_{n}\right) e^{-\alpha_{n+1} t}+\left(s_{n+2}-s_{n+1}\right) e^{-\alpha_{n+2} t}+. .+\left(s_{n+p}-s_{n+p-1}\right) e^{-\alpha_{n+p} t} \\
& =-s_{n} e^{-\alpha_{n+1} t}+s_{n+1} e^{-a_{n+p} t} \\
& +s_{n+1}\left(e^{-a_{n+1} t}-e^{-\alpha_{n+1} t}\right)+\ldots+s_{n+p-1}\left(e^{-a_{n+p-1} t}-e^{-a_{n+\mu} t}\right) \cdot \quad(5)
\end{aligned}
$$

Since, $a_{n+1}<a_{n+2}$, etc., the differences $\left(e^{-a_{n+1} t}-e^{-a_{n+2} t}\right)$, etc., are all positive. Hence if $A$ is the greatest and $B$ the least of the values of $s_{m}$ where $m$ is any integer whatever greater than $n$, 
the sum of those terms in (5) that contain the differences $\left(e^{-a_{n+1} t}-e^{-a_{n+2} t}\right)$, etc., will lie between

$$
\mathbf{A}\left(e^{-a_{n+1} t}-e^{-a_{n+p} t}\right) \text { and } \mathbf{B}\left(e^{-a_{n+1} t}-e^{-a_{n+p} t}\right) \text {. }
$$

If $M$ be some mean value between $A$ and $B$, we may therefore write

$$
M\left(e^{-a_{n+1} t}-e^{-a_{n+p} t}\right) \text {. }
$$

for the sum of the terms in (5) that contain the differences. A, B, M are functions of $n$ but not of $p$.

It is to be noted that the limit for $n$ increasing indefinitely of each of the quantities $A$ and $B$ and therefore of $M$ is $s$, that is

$$
\operatorname{Limit}_{n=\infty} \mathbf{M}=8 \text { - - . }
$$

Equation (5) may now be written

$$
{ }_{p} \mathbf{R}_{n}=\left(M-s_{n}\right) e^{-\alpha_{n+1} t}+\left(s_{n+p}-M\right) e^{-\alpha_{n+p} t} \quad .
$$

and

$$
\psi(t)=\operatorname{Limit}_{p=\infty} \mathbf{R}_{n}=\left(\mathbf{M}-s_{n}\right) e^{-a_{n+1} t}+(s-\mathbf{M}) e^{-a t}
$$

where $a=\operatorname{Limit}_{p=\infty} a_{n+p}$. Usually $a$ is infinite, so that in this case $\psi(t)$ would reduce to the first term.

Again let $s=8_{n}+r_{n}$; then

$$
f(t)-s=\left(\phi(t)-s_{n}\right)+\left(\mathbf{M}-s_{n}\right) e^{-a_{n+1} t}+(s-\mathbf{M}) e^{-a t}-r_{n}
$$

It is easy now to see that equation (3) is true. First, choose $n$ so great, say $n=\mathrm{N}$, that for that and all greater values

$$
\left|\left(M-s_{n}\right) e^{-a_{n+1} t}+(s-M) e^{-a t}-r_{n}\right|<\frac{1}{2} \epsilon .
$$

This choice is possible from (7) and from the fact that the series (1) is convergent. Next, the difference $\left|\phi(t)-s_{\mathrm{N}}\right|$, since it consists of a finite number of terms, can clearly by taking $t$ small enough be made less than $\frac{1}{2} \epsilon$. Hence we can choose $t$ so near zero that

that is

$$
|f(t)-8|<\epsilon
$$

$$
\text { Limit } f(t)=8 \text {. }
$$

The part of the proof that brings in the restriction on the $a$ 's is that which leads to equations (6), (9); the differences

$$
\left(e^{-a_{n+1} t}-e^{-a_{n+2} t}\right) \text {, etc., }
$$

must be positive, or at least all of the same sign. 
Next, instead of $e^{-a_{n} t}$ take a function $\phi_{m}(t)$ such that

(i) Limit $\phi_{m}(t)=1$ for every $m$,

which caincides with the condition $\phi_{m}(0)=1$ if $\phi_{m}(t)$ is continuous up to $t=0$ inclusive.

(ii) $\phi_{1}(t), \phi_{2}(t)$,

is an increasing (or a decreasing) sequence with a finite upper (or lower) limit, $\Phi(t)$.

(iii) $f(t)=\Sigma a_{m} \phi_{m}(t)$ is convergent for $0<t$;

$$
\text { then } \quad \operatorname{Limit}_{t=0} f(t)=s \text {. }
$$

The proof is the same as before. Instead of (6) we have

and instead of $(9)$

$$
\mathbf{M}\left(\phi_{n+1}(t)-\phi_{n+p}(t)\right)
$$

$$
\psi(t)=\left(\mathbf{M}-s_{n}\right) \phi_{n+1}(t)+(s-\mathbf{M}) \Phi(t) \text {. }
$$

